\title{
T1 mapping and T2 mapping at 3T for quantifying the area-at-risk in reperfused STEMI patients
}

Heerajnarain Bulluck ${ }^{1,2,3^{*}+}$, Steven K. White ${ }^{1,2,3+}$, Stefania Rosmini ${ }^{3}$, Anish Bhuva ${ }^{3}$, Thomas A. Treibel ${ }^{3}$, Marianna Fontana ${ }^{3}$, Amna Abdel-Gadir ${ }^{3}$, Anna Herrey ${ }^{3}$, Charlotte Manisty ${ }^{3}$, Simon M. Y. Wan ${ }^{4}$, Ashley Groves ${ }^{4}$, Leon Menezes ${ }^{4}$, James C. Moon ${ }^{2,3}$ and Derek J. Hausenloy ${ }^{1,2,3,5,6}$

\begin{abstract}
Background: Whether T1-mapping cardiovascular magnetic resonance (CMR) can accurately quantify the area-at-risk (AAR) as delineated by T2 mapping and assess myocardial salvage at 3T in reperfused ST-segment elevation myocardial infarction (STEMI) patients is not known and was investigated in this study.

Methods: 18 STEMI patients underwent CMR at 3T (Siemens Bio-graph mMR) at a median of 5 (4-6) days post primary percutaneous coronary intervention using native T1 (MOLLI) and T2 mapping (WIP \#699; Siemens Healthcare, UK). Matching short-axis T1 and T2 maps covering the entire left ventricle (LV) were assessed by two independent observers using manual, Otsu and 2 standard deviation thresholds. Inter- and intra-observer variability, correlation and agreement between the $\mathrm{T} 1$ and $\mathrm{T} 2$ mapping techniques on a per-slice and per patient basis were assessed.

Results: A total of 125 matching T1 and T2 mapping short-axis slices were available for analysis from 18 patients. The acquisition times were identical for the T1 maps and T2 maps. 18 slices were excluded due to suboptimal image quality. Both mapping sequences were equally prone to susceptibility artifacts in the lateral wall and were equally likely to be affected by microvascular obstruction requiring manual correction. The Otsu thresholding technique performed best in terms of inter- and intra-observer variability for both T1 and T2 mapping CMR. The mean myocardial infarct size was $18.8 \pm 9.4 \%$ of the LV. There was no difference in either the mean AAR $(32.3 \pm 11.5 \%$ of the LV versus $31.6 \pm 11.2 \%$ of the $L V, P=0.25)$ or myocardial salvage index $(0.40 \pm 0.26$ versus $0.39 \pm 0.27, P=0.20)$ between the $T 1$ and $T 2$ mapping techniques. On a per-slice analysis, there was an excellent correlation between T1 mapping and T2 mapping in the quantification of the AAR with an $R^{2}$ of $0.95(P<0.001)$, with no bias (mean \pm 2 SD: bias $0.0 \pm 9.6 \%$ ). On a per-patient analysis, the correlation and agreement remained excellent with no bias $\left(R^{2} 0.95, P<0.0001\right.$, bias $\left.0.7 \pm 5.1 \%\right)$.
\end{abstract}

Conclusions: T1 mapping CMR at 3T performed as well as T2 mapping in quantifying the AAR and assessing myocardial salvage in reperfused STEMI patients, thereby providing an alternative CMR measure of the the AAR.

Keywords: Area-at-risk, Myocardial salvage, Cardiovascular MR, T1 mapping, T2 mapping, ST-elevation myocardial infarction, Primary percutaneous intervention

\footnotetext{
* Correspondence: h.bulluck@gmail.com

${ }^{\dagger}$ Equal contributors

'The Hatter Cardiovascular Institute, Institute of Cardiovascular Science,

University College London, London WC1E 6HX, UK

${ }^{2}$ The National Institute of Health Research University College London

Hospitals Biomedical Research Centre, London, UK

Full list of author information is available at the end of the article
} reproduction in any medium, provided you give appropriate credit to the original author(s) and the source, provide a link to the Creative Commons license, and indicate if changes were made. The Creative Commons Public Domain Dedication waiver (http://creativecommons.org/publicdomain/zero/1.0/) applies to the data made available in this article, unless otherwise stated. 


\section{Background}

Despite timely myocardial reperfusion by primary percutaneous coronary intervention (PPCI), patients presenting with an acute ST-segment elevation myocardial infarction (STEMI) still experience significant morbidity and mortality [1-3]. New cardioprotective therapies are therefore required to reduce myocardial infarct (MI) size, in order to preserve left ventricular (LV) ejection fraction and prevent the onset of heart failure. The assessment of the efficacy of novel cardioprotective therapies requires the accurate quantification of the area-at-risk (AAR), as this enables the measurement of the myocardial salvage index (AAR subtract MI size/AAR), a more sensitive measure of cardioprotective effectiveness than a reduction in absolute MI size or MI size as a percentage of the LV alone $[4,5]$.

In this regard, T2-weighted (short tau inversion recovery) cardiovascular magnetic resonance (CMR) of myocardial edema in the first few days following PPCI has emerged as a promising technique for retrospectively quantifying the AAR in reperfused STEMI patients [6, 7]. This approach, however, does have its limitations which include a low contrast-to-noise ratio, susceptibility to blood pool and motion artifacts, and signal drop-out. Some of these can be overcome using T2-mapping CMR, whch has emerged as a more robust surrogate marker to delineate the AAR in this setting $[8,9]$. However, recent studies have found that in addition to reducing MI size, certain cardioprotective therapies such as ischemic postconditioning [10] and remote ischemic conditioning [11, 12] also decreased the extent of myocardial edema as delineated by $\mathrm{T} 2$ mapping and T2-weighted CMR, resulting in an underestimation of the AAR with this approach.

Recently, native T1-mapping CMR (referred to as T1-mapping or T1 map throughout the manuscript for simplicity) has been found to be superior to T2weighted CMR in detecting myocardial edema in the context of acute myocarditis [13] and acute myocardial infarction [14]. T1-mapping CMR has recently been reported to accurately quantify the AAR in the canine heart subjected to acute myocardial infarction [15]. Langhans et al. [16] found that AAR by T1 and T2 mapping CMR at $1.5 \mathrm{~T}$ correlated well with that obtained with myocardial SPECT. In healthy volunteers, von Knobelsdorff-Brenkenhoff et al. [17] have recently demonstrated feasibility and provided reference values for $\mathrm{T} 1$ and $\mathrm{T} 2$ mapping at 3T, but the role of these CMR sequences at $3 \mathrm{~T}$ has not been investigated in the setting of acute MI. Whether T1-mapping CMR can quantify the AAR at 3T, to our knowledge, has not been directly compared to T2 mapping in reperfused STEMI patients, and was investigated in the current study.

\section{Methods}

\section{Patient population}

18 PPCI-treated STEMI patients were recruited over a 7month period from one UK center. The main exclusion criteria were previous $\mathrm{MI}$ and standard contraindications to CMR (significant claustrophobia, severe allergy to gadolinium chelate, estimated glomerular filtration rate $<30 \mathrm{~mL} /$ $\mathrm{min} / 1.73 \mathrm{~m}^{2}$, presence of ferromagnetic implants). All eligible patients provided informed written consent and the local ethics committee (London - Harrow) approved all study-related procedures. The patients recruited in this study formed part of a cohort of patients included in a recently conducted hybrid simultaneous Positron Emission Tomography/MR study [18].

\section{CMR}

Patients underwent CMR at a median of 5 (4-6) days post-PPCI using a 3T MR scanner (Biograph mMR; Siemens Healthcare, Erlangen, Germany). The MR imaging protocol included cine imaging for function, followed by T1 maps and T2 maps for AAR and Late Gadolinium Enhancement (LGE) for MI size. T1 maps and T2 maps (Works in Progress, software \#699, Siemens Healthcare, Frimley, UK) were acquired as previously described [17]. For T1 maps by SSFP-based Modified LookLocker Inversion Recovery (MOLLI) technique, imaging parameters were: repetition time $=2.6-2.7 \mathrm{~ms}$; echo time $=1.0-1.1 \mathrm{~ms}$; flip angle $=35^{\circ}$; matrix $=256 \times 144$; slice thickness $6 \mathrm{~mm}$. Images were acquired at different inversion times (5-3-3 modified MOLLI protocol to reduce heart rate variability by acquiring 5 images after the first inversion pulse, followed by a 3 heartbeat pause and then acquiring the last 3 images after the second inversion pulse [19]) and registered prior to a non-linear least-square curve fitting to generate a pixel-wise coloured T1 map. For T2 maps, 3 single shot images were acquired at different T2-preparation times ( $0 \mathrm{~ms}, 24 \mathrm{~ms}$, and $55 \mathrm{~ms}$, respectively) and imaging parameters were: repetition time $=2.4 \mathrm{~ms}$; echo time $=1 \mathrm{~ms}$; flip angle $=70^{\circ}$; repetition time $=3 \times \mathrm{R}-\mathrm{R}$ interval; acquisition matrix $116 \times 192$; slice thickness $6 \mathrm{~mm}$; field of view adjusted as per subject size. Motion correction and fitting were performed to estimate coefficients of the decay function, which were then used to estimate the T2 times. An in-built specific colour look-up table was then used to derive the coloured T2 maps.

The T1 and T2 maps were acquired to match the short-axis cines to cover the entire left ventricle. Gadolinium contrast (Gadoterate meglumine, gadoliniumDOTA, marketed as Dotarem, Guerbet S.A., Paris, France) was administered at a dose of $0.1 \mathrm{mmol} / \mathrm{kg}$. LGE was performed using segmented two-dimensional inversion-recovery turbo fast low-angle shot LGE sequences (repetition time $=864 \mathrm{~ms}$; echo time $=$ $1.56 \mathrm{~mm}$; acquisition matrix $=123 \times 256$; inversion time 
$=400-500 \mathrm{~ms}$, flip angle $=20^{\circ}$; slice thickness $8 \mathrm{~mm}$ ) 10-15 min after single bolus contrast agent injection and short-axis slices of the entire left ventricle were acquired to match the T1 maps and T2 maps (Fig. 1).

\section{Imaging analysis}

Quantification of LV volumes, LV mass, LV ejection fraction and MI size were performed using CVI42 software (Version 5.1.0[280], Calgary, Canada). MI size was quantified following manual delineation of the endocardium and epicardium of short axis slices and using 5 standard deviations (SD) threshold above the mean remote myocardium [20]. Transmural extent of LGE was also quantified using 100 chords for each short axis slice and averaging the mean transmural extent for each segment using the modified 16 segment American Heart Association (AHA) model [21]. Segments were assigned an LGE score of either " 0 " for no LGE and " 1 " for the presence of LGE. Mean segmental T1 and T2 values were also automatically generated for the modified 16 segment AHA model using CVI42 after manually drawing the endocardial and epicardial borders on all short axis slices. The time taken to acquire the 2 mapping sequences for each patient (LV coverage from base to apex) were recorded. AAR by T1 mapping and T2 mapping were quantified using an in-house macro written in ImageJ (Version $1.45 \mathrm{~s}$, National Institute of Health, USA). The endocardium and epicardium borders of matching LV short axis T1 maps and T2 maps were manually segmented (excluding the papillary muscles) to obtain the myocardium volume. Two experienced observers performed all subsequent quantification on the pre-segmented images blindly and independently, and one of the observers performed the analysis twice,
3 months apart. The affected myocardium on the T1 maps and T2 maps were quantified using 3 analytical techniques namely manual delineation, 2SD above the mean remote normal myocardium, and the automated Otsu detection method (Otsu technique) [22]. In brief, the Otsu technique uses an algorithm to automatically divide the signal intensity histogram into normal and enhanced. An exhaustive search for values that minimize the intraclass variance between two populations of signal intensities is used to establish the threshold [23]. Analysis was performed for all the slices for each patient to obtain the "enhanced" myocardium as a percentage of the whole LV. Regions-of-interest were drawn within the AAR (avoiding areas of MVO), and within the remote myocardium to obtain representative $\mathrm{T} 1$ and $\mathrm{T} 2$ values at $3 \mathrm{~T}$ CMR. Manual correction was performed for areas of pseudonormalization within the MI zone (corresponding to areas of MVO) and areas of hyperenhancement due to any obvious blood pool or pericardial partial voluming and off-resonance artifacts in the remote myocardium. All slices were visually assessed by the 2 experienced observers and those with significant partial voluming and susceptibility or motion artifacts overlapping with the affect myocardium were excluded by consensus. In cases of doubt or disagreement, the raw images were used to decide whether that slice was to be excluded or not as previously described by von Knobelsdorff-Brenkenhoff et al. [17].

\section{Statistical analysis}

Statistical analysis was performed using SPSS (Version 22, IBM Corporation, Illinois, USA) and MedCalc (Version 15.6.1, MedCalc Software bvba, Ostend, Belgium). Continuous data was expressed as mean $\pm \mathrm{SD}$ or median (interquartile range). Categorical data was reported as frequencies and percentages. Both per-slice and per-patient comparison

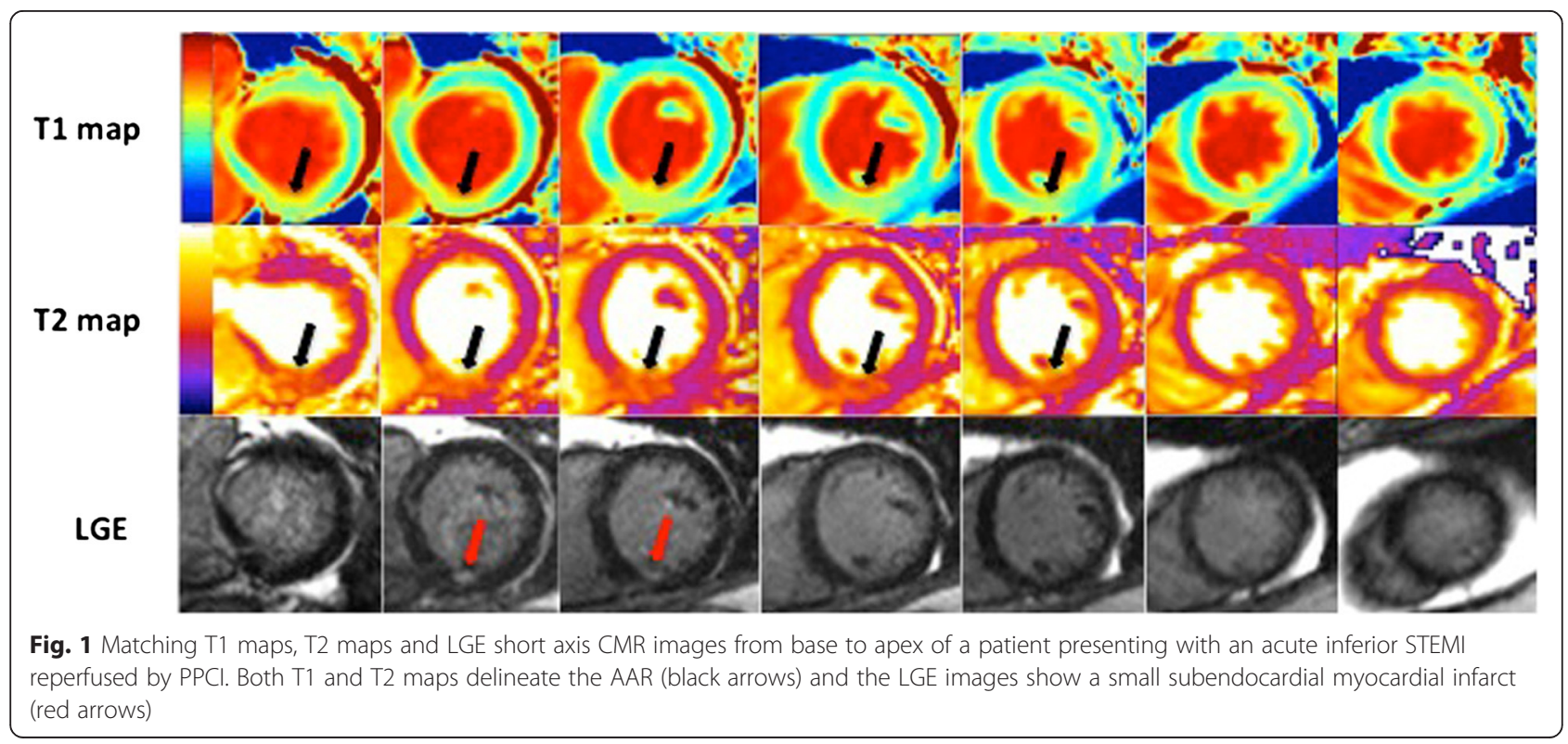


was performed. Paired student t-tests and Wilcoxon Rank sum test were performed to compare mean or median between paired groups. Pearson's correlation coefficient expressed as its square $\left(R^{2}\right)$ was used to assess intermethod correlation. A linear regression analysis was performed to obtain the regression slope and its $95 \%$ confidence interval to compare against the reference line with a slope of 1 which would represent AAR by T1 and T2 to be identical. Bland-Altman analysis was performed to assess agreement and bias detection between methods and presented as average difference $\pm 2 \mathrm{SD}$. Inter-observer and intra-observer variability was assessed using intra-class correlation coefficient (ICC) and mean difference between interobserver and intraobserver measurements of $\mathrm{T} 1$ and $\mathrm{T} 2 \mathrm{AAR} \pm \mathrm{SD}$. Receiver Operating Characteristic (ROC) analysis was performed to provide cut-off values for T1 and $\mathrm{T} 2$ for detecting acute myocardial necrosis defined by an LGE score of 1 . ROC curves were compared for statistical difference using the method described by Delong et al. [24]. All statistical tests were two-tailed, and Pvalues of less than 0.05 were considered statistically significant.

\section{Results}

The patients' demographics, coronary angiographic and CMR characteristics are detailed in Table $1.83 \%$ were male and the mean age was $58 \pm 10$ years. Two thirds of the patients presented with a left anterior descending (LAD) territory STEMI and one third with a right coronary artery (RCA) territory infarct. The median onset to balloon time was $292(116-800)$ minutes. Figure 2 shows examples of T1 maps, T2 maps and LGE in 3 different patients.

\section{Quality and acquisition times of T1 and T2 maps}

In total, 125 pairs of T1 maps and T2 maps of the LV short axis from base to apex were acquired. The acquisition times for T1 maps and T2 maps per patient were similar (T1 maps: $5.6 \pm 1.9 \mathrm{~min}$, T2 maps: $5.5 \pm 2.0 \mathrm{~min}$, $P=0.89$ ). 18 pairs of images were excluded due to suboptimal image quality (15 apical short-axis slices and 3 basal short-axis slices including the LVOT which had significant partial voluming). Both T1 and T2 CMR were equally prone to susceptibility artifacts in the lateral wall. As we did not have any patients with a circumflex territory infarction, these artifacts were remote from the AAR and the slices (10/107 slices, $9 \%$ ) were kept for analysis and manual correction was required. 27 slices had normal T1 and T2 values and 80 slices had abnormal T1 and T2 values (using 2SD from the remote myocardium as a reference). Both $\mathrm{T} 1$ and $\mathrm{T} 2$ maps were equally likely to be affected by MVO requiring manual correction to include the core as part of the AAR and occurred in 13/18 patients, 45/107 slices of the T2 maps, and $42 / 107$ of the T1 maps.
Table 1 Patients' demographics, coronary angiographic and CMR characteristics. This table provides the demographic details, coronary angiographic and CMR characteristics of the study population

\begin{tabular}{|c|c|}
\hline Details & Number \\
\hline Number of patients & 18 \\
\hline Male (\%) & $15(83)$ \\
\hline Age $\pm S D$ (years) & $58 \pm 10$ \\
\hline Hypertension (\%) & $5(28)$ \\
\hline Smoking (\%) & $9(50)$ \\
\hline Dyslipidemia (\%) & $3(17)$ \\
\hline Chest pain onset to balloon time (minutes) & $292(116-800)$ \\
\hline \multicolumn{2}{|l|}{ Infarct artery and location (\%) } \\
\hline LAD & $12(67)$ \\
\hline Proximal/ Mid/ Distal & $5(42) / 6(50) / 1(8)$ \\
\hline RCA & $6(33)$ \\
\hline Proximal/ Mid/ Distal & $3(50) / 2(33) / 1(17)$ \\
\hline \multicolumn{2}{|l|}{ Pre-PPCI TIMI flow (\%) } \\
\hline $0 / 1 / 2 / 3$ & $13(72) / 4(22) / 1(6) / 0(0)$ \\
\hline \multicolumn{2}{|l|}{ Post-PPCI TIMI flow (\%) } \\
\hline $0 / 1 / 2 / 3$ & $1(6) / 0(0) / 2(12) / 15(82)$ \\
\hline Single vessel disease & $13(72)$ \\
\hline Double vessel disease & $5(28)$ \\
\hline Days from $\mathrm{PPCl}$ to $\mathrm{CMR}$ & $5(4-6)$ \\
\hline Left ventricular ejection fraction (\%) & $49 \pm 11$ (Normal range 58-76) \\
\hline End diastolic volume (ml) & $135 \pm 21$ (Normal range 113-196) \\
\hline Left ventricular mass (g) & $151 \pm 50$ (Normal range 107-184) \\
\hline Presence of MVO (\%) & $8(44)$ \\
\hline Infarct size by LGE, (\% LV volume) & $18.8 \pm 9.4$ \\
\hline AAR by T1-mapping, (\% LV volume) & $32.3 \pm 11.5$ \\
\hline AAR by T2-mapping, (\% LV volume) & $31.6 \pm 11.2$ \\
\hline
\end{tabular}

$P P C l$ primary percutaneous coronary intervention, $L A D$ left anterior descending artery, $R C A$ right coronary artery, TIMI thrombolysis in myocardial infarction, $L V$ left ventricle, $S D$ standard deviation, MVO microvascular obstruction, $L G E$ Late Gadolinium Enhancement, $A A R$ area-at-risk

\section{Inter and intra-observer variability}

The AAR by the 3 techniques (manual/Otsu/2SD) for T2 mapping were $31.8 \pm 11.7 \%, 31.6 \pm 11.2 \%$ and $38.7 \pm 15 \%$ and for $\mathrm{T} 1$ mapping were $32.0 \pm 11.5 \%$, $32.3 \pm 11.5 \%$ and $38.4 \pm 13.6 \%$ respectively. The ICC for intra-observer and inter-observer variability of the 3 analytical techniques were excellent, both for T1 and T2 mapping and was highest for the Otsu technique. The 2SD technique had the largest differences both for intra-observer and inter-observer measurements for both mapping techniques. These findings are summarized in Table 2. The 2SD technique overestimated the AAR compared to manual delineation (as the reference standard) but there was no difference between Otsu and manual delineation for both $\mathrm{T} 1$ and $\mathrm{T} 2$ 


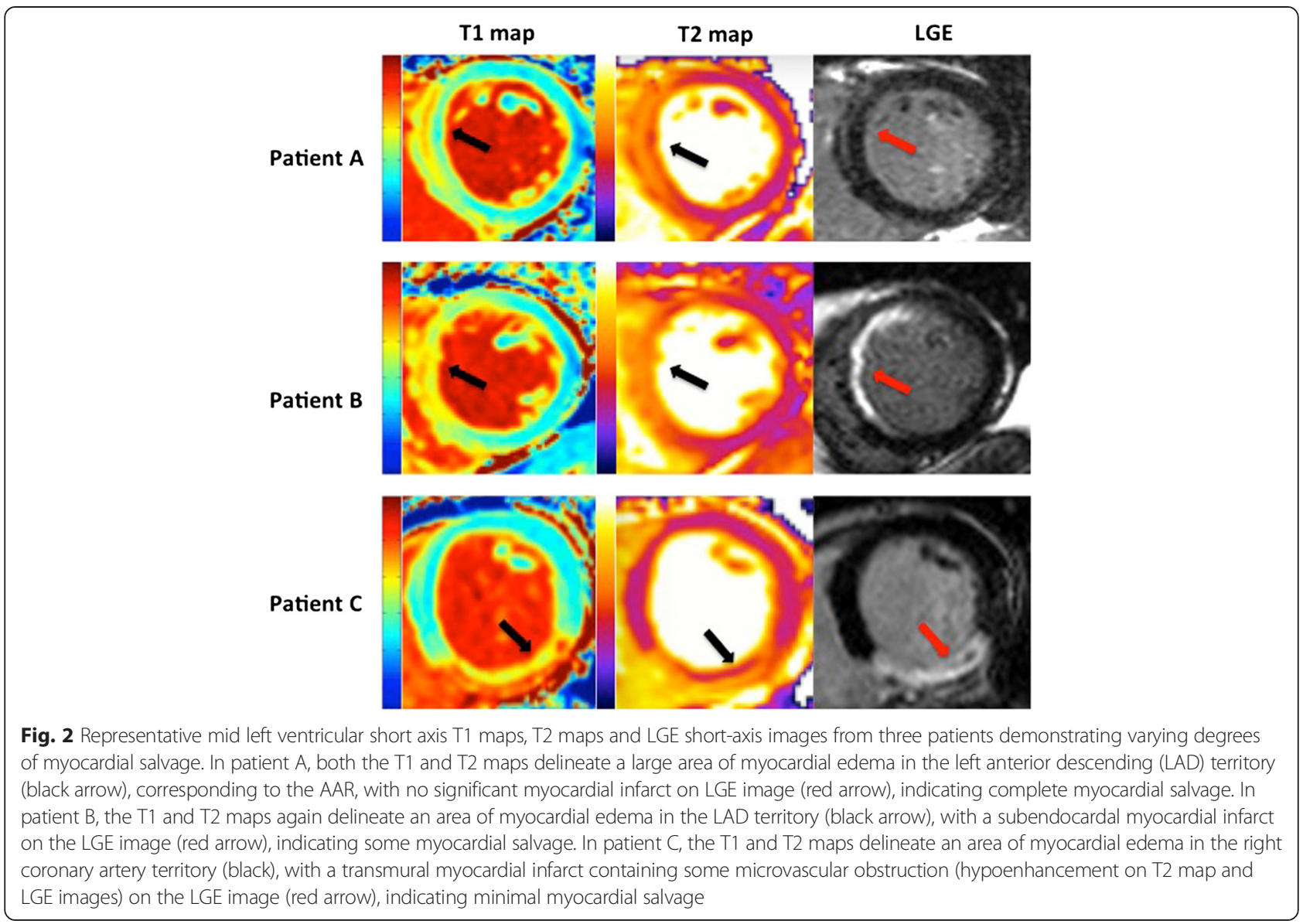

mapping (Fig. 3). The Otsu derived T1 and T2 AAR was therefore used for the analysis below.

\section{Quantification of the AAR by T1 mapping}

On a per-slice analysis, there was an excellent correlation between the T1 mapping and T2 mapping with an $\mathrm{R}^{2}$ of 0.95 and a regression slope 0.97 (95\% CI 0.93 1.01). There was no bias on Bland Altman analysis (mean \pm 2 SD: bias $0.0 \pm 9.6 \%$ ) as illustrated in Fig. 4a. On a per-patient analysis, the correlation and agreement remained excellent with no bias $\left(R^{2} 0.95\right.$, regression slope 0.95 (95 \% CI $0.84-1.07$ ), bias $0.7 \pm 5.1 \%$; Fig. 4b). The mean AAR (expressed as a \% of the LV) quantified by T1 mapping was similar to that by T2 mapping (32.3 \pm $11.5 \%$ of the LV, range 6 to $52 \%$ by T1 mapping, versus $31.6 \pm 11.2 \%$ of the LV, range $5-48 \%$ by T2 mapping, $P=0.25)$.

\section{MI size and myocardial salvage}

The mean MI size by LGE was $18.8 \pm 9.4 \%$ of the LV, (range $2.0-34.0 \%$ ). Myocardial salvage (AAR subtract the MI size) was $12.8 \pm 10.0 \%$ of LV (range $0-42.0 \%$ ) by T2 mapping. The myocardial salvage index (myocardial salvage/AAR) was $0.40 \pm 0.26$ (range $0-0.89$ ). There were no difference in either myocardial salvage $(12.8 \pm 10.0 \%$ of LV by T 2 mapping versus $13.5 \pm 10.4 \%$ of LV by T1 mapping, $P=0.25$ ) (Fig. 3) or the myocardial salvage index $(0.40 \pm 0.26$ by T2 mapping versus $0.39 \pm 0.27$ by T1 mapping, $P=0.20$ ) between the 2 mapping techniques.

\section{$\mathrm{T} 1$ and $\mathrm{T} 2$ values in the AAR and remote myocardium}

The T1 and T2 values in the AAR was significantly higher than those in the remote myocardium (T1 AAR: $1524 \pm$ $116 \mathrm{~ms}$, T1 remote myocardium: $1163 \pm 78 \mathrm{~ms}, P<0.001$; T2 AAR: $72 \pm 7 \mathrm{~ms}$, T2 remote myocardium: $46 \pm 3 \mathrm{~ms}$, $P<0.001)$.

\section{Diagnostic performance of $\mathrm{T} 1$ and $\mathrm{T} 2$ mapping to detect acute myocardial necrosis}

Both $\mathrm{T} 1$ and $\mathrm{T} 2$ mapping performed well to detect acute myocardial necrosis delineated by the presence of LGE as illustrated in the ROC curves in Fig. 5. The area under the curve (AUC) was $0.87 \pm 0.02$ for $\mathrm{T} 1$ and $0.86 \pm 0.02$ for T2, $P=0.96$. A T1 value of $>1249 \mathrm{~ms}$ had a sensitivity of $83 \%$ and specificity of $80 \%$ and a T2 value of $>52 \mathrm{~ms}$ had a sensitivity of $85 \%$ and specificity of $82 \%$ to detect acute myocardial necrosis defined by an LGE score of 1 . 
Table 2 Intra-observer and inter-observer variability of the areaat-risk by $\mathrm{T} 1$ and T2. Intra-observer and inter-observer variability of the area-at-risk by $\mathrm{T} 1$ and $\mathrm{T} 2$. This table provides the intra-class correlation coefficient (ICC) and mean difference \pm SD for the inter-observer and intra-observer measurements of $\mathrm{T} 1$ mapping and T2 mapping using 3 analytical techniques for inter-observer and intra-observer variability

\begin{tabular}{|c|c|c|c|}
\hline & ICC (95\% Cl) & Mean difference \pm SD (\%) & $P$ \\
\hline & \multicolumn{3}{|c|}{ Intra-observer variability $(n=107)$} \\
\hline \multicolumn{4}{|c|}{ T1 mapping } \\
\hline Manual & $0.961(0.943-0.973)$ & $1.5 \pm 7.1$ & $0.04^{*}$ \\
\hline $2 S D$ & $0.948(0.917-0.966)$ & $2.6 \pm 7.7$ & $0.001^{*}$ \\
\hline Otsu & $0.989(0.984-0.993)$ & $0.7 \pm 3.2$ & $0.03^{*}$ \\
\hline \multicolumn{4}{|c|}{ T2 mapping } \\
\hline Manual & $0.951(0.928-0.966)$ & $0.8 \pm 6.4$ & 0.18 \\
\hline $2 S D$ & $0.965(0.942-0.978)$ & $2.4 \pm 6.4$ & $0.001^{*}$ \\
\hline \multirow[t]{2}{*}{ Otsu } & $0.996(0.995-0.998)$ & $0.2 \pm 1.9$ & 0.24 \\
\hline & \multicolumn{3}{|c|}{ Inter-observer variability $(n=107)$} \\
\hline \multicolumn{4}{|c|}{ T1 mapping } \\
\hline Manual & $0.980(0.972-0.987)$ & $0.3 \pm 4.3$ & 0.52 \\
\hline $2 S D$ & $0.948(0.925-0.964)$ & $3.7 \pm 7.2$ & $0.001^{*}$ \\
\hline Otsu & $0.993(0.990-0.995)$ & $0.2 \pm 2.6$ & 0.55 \\
\hline \multicolumn{4}{|c|}{ T2 mapping } \\
\hline Manual & $0.964(0.947-0.975)$ & $0.3 \pm 6.1$ & 0.59 \\
\hline $2 S D$ & $0.960(0.914-0.978)$ & $3.5 \pm 6.5$ & $0.001^{*}$ \\
\hline Otsu & $0.993(0.989-0.995)$ & $0.7 \pm 2.6$ & $0.008^{*}$ \\
\hline
\end{tabular}

*denotes significant statistical difference with $\mathrm{P}<0.05$

\section{Discussion}

This is the first clinical study to demonstrate T1 mapping CMR at 3T can accurately quantify the AAR delineated by $\mathrm{T} 2$ mapping CMR in reperfused STEMI patients. There was an excellent correlation and agreement between T1 and T2 mapping in delineating the AAR. This data confirms the findings of the pre-clinical study by Ugander et al. [15] in the reperfused canine heart. Both T1 and T2 mapping CMR were equally prone to susceptibility artifacts at $3 \mathrm{~T}$ and equally affected by MVO. There was no difference in the acquisition times on a per-patient basis.

Unlike the canine model from Ugander et al. [15], we included patients with different ischemic times (chest pain onset to PPCI times), different degrees of myocardial salvage, LAD as well as RCA infarcts, and with the presence of MVO. The current study therefore reinforces emerging evidence that T1 mapping performs as well as $\mathrm{T} 2$ mapping at $3 \mathrm{~T}$ and therefore provides us with an additional tool to quantify the AAR.

This is the first study to compare the diagnostic performance of $\mathrm{T} 1$ mapping against $\mathrm{T} 2$ mapping at $3 \mathrm{~T}$ to detect acute myocardial necrosis and the results are in keeping with the previous study by Dall'Armellina et al. [14] in which T1 mapping by ShMOLLI was compared with T2 weighted imaging at 3T. We performed an indirect comparison of the 2 ROC curves by the MOLLI protocol used in our study and ShMOLLI T1 mapping from their study [14] and found that there was no difference between the 2 AUCs (comparison of independent ROC curves using MedCalc Version 15.6.1 Software bvba, AUC of $0.87 \pm 0.02$ by MOLLI T1 mapping versus AUC of $0.90 \pm 0.01$ by ShMOLLI mapping, difference of $0.03 \pm 0.02$, z statistic $1.34, P=0.17)$.

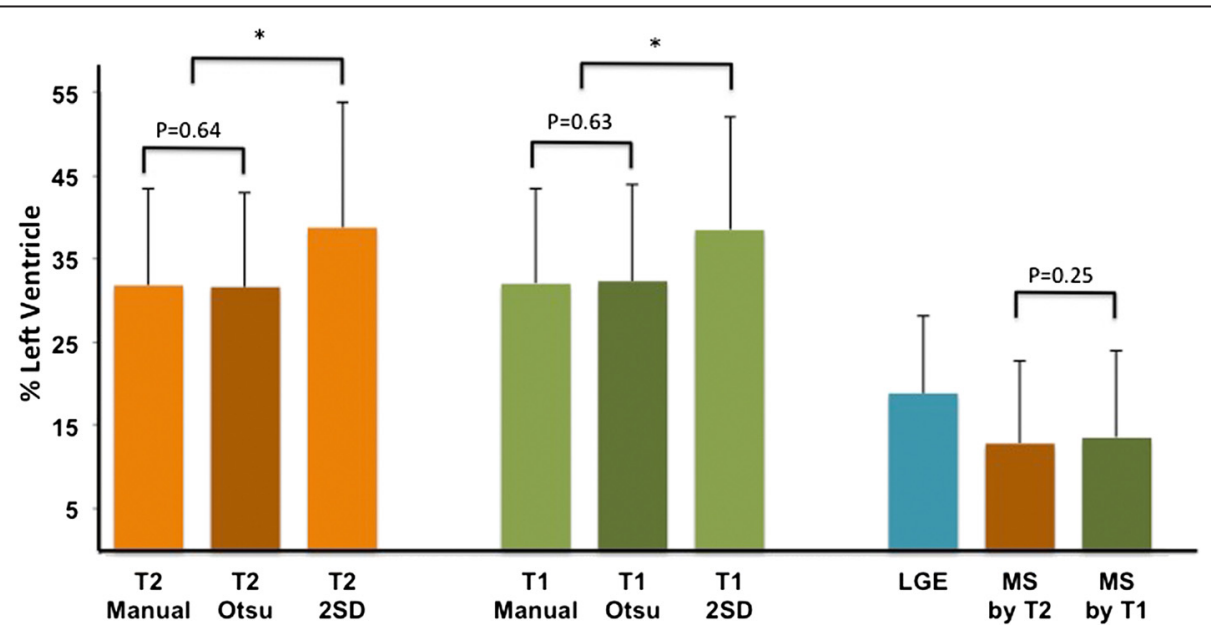

Fig. 3 Performance of three different thresholding techniques for delineating the AAR on T1 and T2 maps. The AAR by the 2 standard deviation (2SD) technique was significantly larger than that delineated by the manual and Otsu thresholding techniques. There was no difference between the manual and Otsu techniques for both T1 and T2 mapping in delineating the AAR. ${ }^{*}$ denotes significant statistical difference with $P<0.001$ 


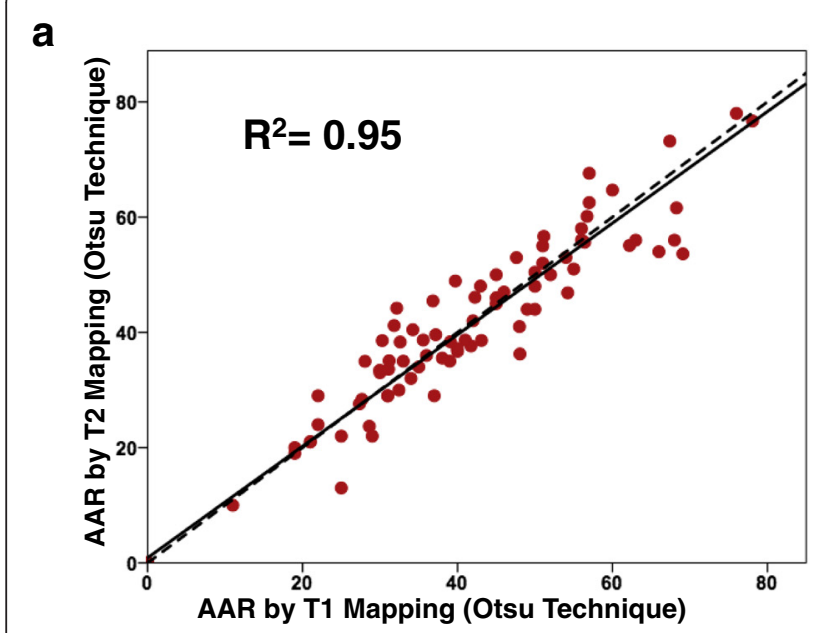

b
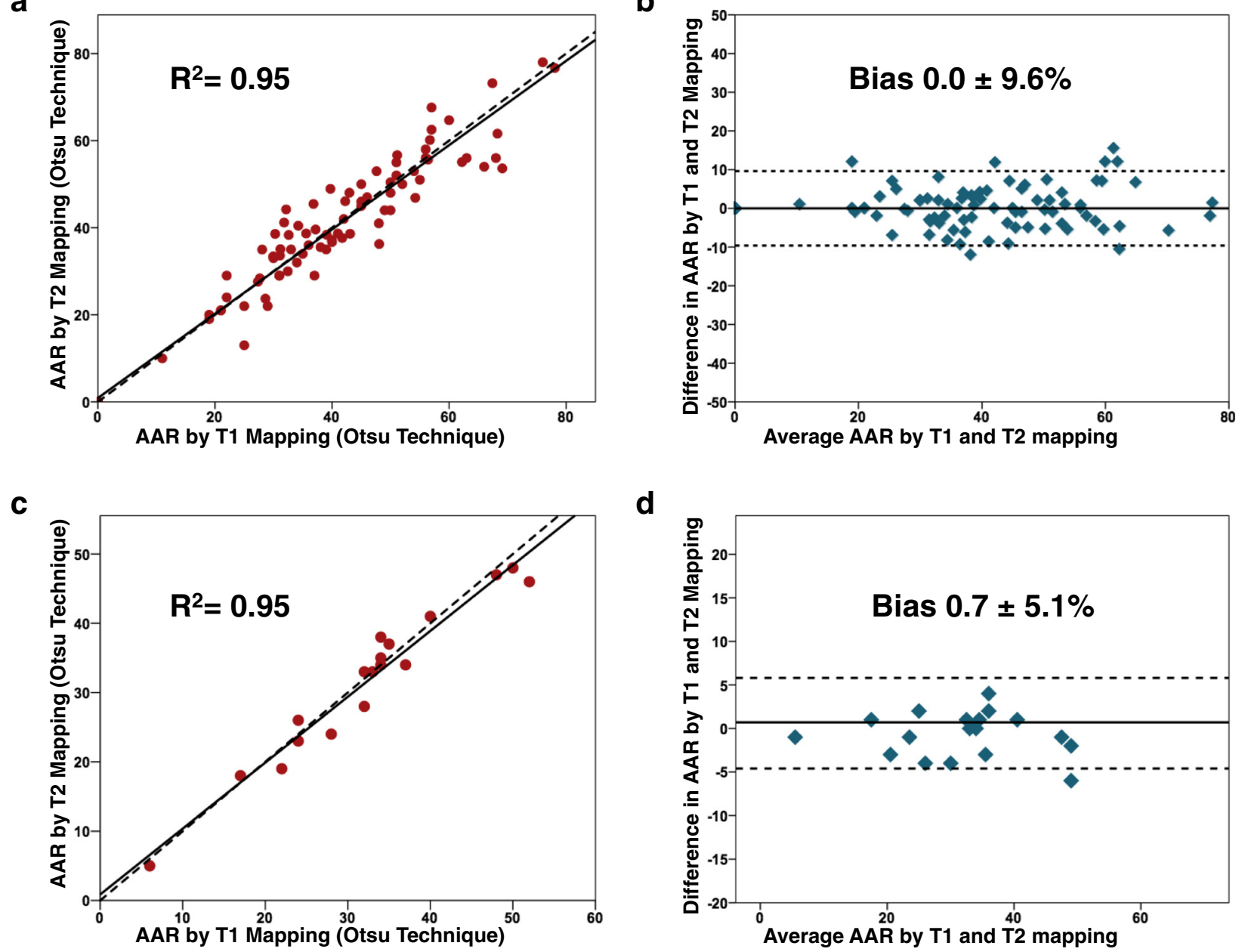

d

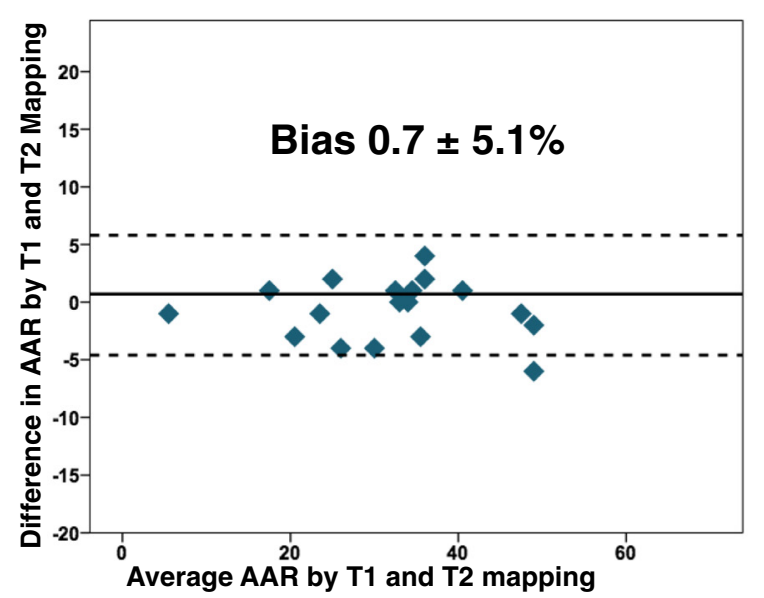

Fig. 4 Correlation and agreement between T1 and T2 mapping to delineate the AAR. Both on a per-slice (a and $\mathbf{b}$ ) and per-patient analysis (c and $\mathbf{d}$ ), there was an excellent correlation and agreement between T1 and T2 mapping technique to delineate the AAR. The interrupted lines in a and $\mathrm{c}$ represent reference lines with a slope of 1

Langhans et al. [16] recent looked at the reproducibility of the AAR by $\mathrm{T} 1$ and $\mathrm{T} 2$ mapping against SPECT in 14 patients with reperfused STEMI at 1.5 T. Although good correlations with SPECT were reported, direct comparison between the two mapping techniques was not performed in terms of per slice correlation, agreement and inter and intra-observer variability.

Optimal thresholding technique for T1 and T2 mapping We found that the Otsu thresholding method performed best with excellent inter-observer and intra-observer variability for both mapping techniques. The algorithm automatically divides a signal intensity histogram into two classes requires minimal user input compared to manual delineation, SD thresholding and full width half maximum techniques. It automatically calculates an optimal threshold [22] and has previously been shown to be more accurate and reproducible for quantifying acute MI size by CMR. [25].

\section{Which to choose: T1 or T2 mapping?}

Currently, it would appear that T1 and T2 mapping could be used interchangeably to assess the AAR in reperfused STEMI patients. In studies investigating post-contrast T1 and extracellular volume fraction in acute myocardial infarction patient, there is the possibility of shortening the scanning time by omitting T2 maps as the T1 maps would be available for AAR quantification. However, T1 mapping may not be suitable in acute myocardial infarction patients who also have a chronic infarct in the remote myocardium. T1 mapping CMR has recently been shown to identify chronic infarct with high diagnostic accuracy [26] in a canine model, and using this technique in these patients would require taking into account areas of chronic infarct in the remote myocardium. Our study excluded patients 


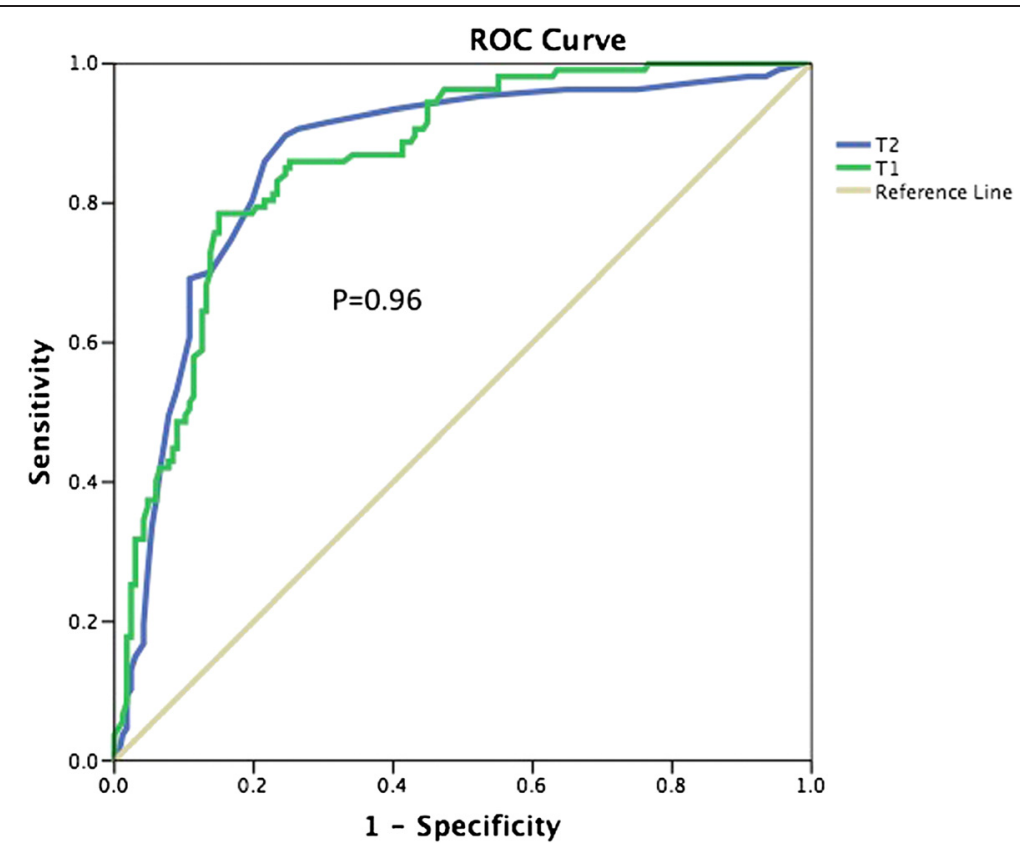

Fig. 5 ROC curve showing the diagnostic performance of T1 mapping against T2 mapping to detect acute myocardial necrosis. Both T1 and T2 mapping performed equally well to detect acute myocardial necrosis. The AUC was $0.87 \pm 0.02$ for T1 and $0.86 \pm 0.02$ for T2, $P=0.96$

with previous infarct and therefore we cannot comment on the performance of T1 mapping over T2 mapping in patients with co-existing chronic infarcts. From an MR physics point of view, these two techniques are assessing different properties of the myocardium and may explain the limit of agreement of around $10 \%$ on a per-slice comparison and of around $5 \%$ on a per-patient comparison and more work remains to be done to establish the advantage of one mapping sequence over the other.

\section{Potential future direction}

Certain cardioprotective therapies such as ischemic postconditioning [10] and remote ischaemic conditioning (using transient arm or leg ischaemia and reperfusion) $[11,12]$ has been shown not only to reduce MI size, but also the extent of edema delineated by $\mathrm{T} 2$ mapping and T2-weighted imaging leading to an underestimate of the AAR in reperfused STEMI patients. Whether the AAR delineated by $\mathrm{T} 1$ mapping is also affected by these therapeutic interventions needs to be investigated. It is currently believed that the AAR delineated by T2-weighted imaging is maximum and constant within the first week following an acute myocardial infarction [27, 28]. Whether the T1 signal remains stable for a longer period of time remains to be tested. A recent pre-clinical study using a porcine model of acute MI has suggested that myocardial edema delineated by T2 CMR may vary over the first week with 2 phases of edema [29]. Whether this is present in reperfused STEMI patients and whether it is apparent with T1 mapping is unknown.

\section{Limitations}

Although, we only included a small number of patients, we did include a range of ischemic times and performed detailed slice-per-slice comparisons, including inter and intra-observer performance. Unfortunately we did not recruit any patients with a circumflex territory myocardial infarction as it would have been interesting to assess the effect of off-resonance artifacts in the lateral wall in those patients with lateral wall MI on both mapping techniques. Our study was performed at $3 \mathrm{~T}$ and whether T1 mapping performs as well as T2 mapping in quantifying the AAR at $1.5 \mathrm{~T}$ in the clinical setting remains to be determined.

\section{Conclusions}

We have shown for the first time that T1 mapping CMR at $3 \mathrm{~T}$ can accurately quantify the AAR delineated by T2 mapping in reperfused STEMI patients. However, further work is needed to replicate these findings at $1.5 \mathrm{~T}$, assess the dynamic change of $\mathrm{T} 1$ over time compared to $\mathrm{T} 2$, and determine whether the AAR delineated by $\mathrm{T} 1$ mapping is affected by cardioprotective therapies in a similar manner to T2 mapping.

\section{Abbreviations}

PPCI: Primary percutaneous coronary intervention; STEMI: ST-segment elevation myocardial infarction; MI: Myocardial infarction; AAR: Area-at-risk;

CMR: Cardiovascular Magnetic Resonance; MOLLI: Modified Look-Locker Inversion Recovery; LGE: Late Gadolinium Enhancement; LV: Left ventricle or ventricular; MVO: Microvascular obstruction; AHA: American Heart Association; ROC: Receiver Operating Characteristic; AUC: Area under the curve; SD: Standard deviation; LAD: Left anterior descending artery; RCA: Right coronary artery. 


\section{Competing interests}

The authors declare that they have no competing interests.

\section{Authors' contributions}

1) Substantial contributions to conception and design, or acquisition of data, or analysis and interpretation of data: HB, SKW, DJH. 2) Involved in drafting the manuscript or revising it critically for important intellectual content: $H B$, SKW, SR, AB, TAT, MF, AAG, AH, CM, SMYW, AG, LM, JCM, DJH. 3) Given final approval of the version to be published; HB, SKW, SR, AB, TAT, MF, AAG, AH, CM, SMYW, AG, LM, JCM, DJH. 4) Agreed to be accountable for all aspects of the work in ensuring that questions related to the accuracy or integrity of any part of the work are appropriately investigated and resolved: HB, SKW, DJH. All authors read and approved the final manuscript.

\section{Acknowledgements}

We express our gratitude to the staff and patients at the UCLH Heart Hospital and Peter Weale for providing us the investigational sequence for T1 and T2 mapping under a research collaboration agreement with Siemens Healthcare. This work was supported by the British Heart Foundation (FS/10/039/28270), the Rosetrees Trust, and the National Institute for Health Research University College London Hospitals Biomedical Research Centre. SKW is supported by British Heart Foundation Clinical Research Training Fellowship (grant number FS/10/72/28568).

\section{Author details}

${ }^{1}$ The Hatter Cardiovascular Institute, Institute of Cardiovascular Science, University College London, London WC1E 6HX, UK. ${ }^{2}$ The National Institute of Health Research University College London Hospitals Biomedical Research Centre, London, UK. ${ }^{3}$ The Heart Hospital, University College London Hospital, London, UK. ${ }^{4}$ UCL Institute of Nuclear Medicine, University College London Hospital, London, UK. ${ }^{5}$ Cardiovascular and Metabolic Disorders Program, Duke-National University of Singapore, Singapore, Singapore. ${ }^{6}$ National Heart Research Institute Singapore, National Heart Centre Singapore, Singapore, Singapore.

\section{Received: 14 April 2015 Accepted: 16 July 2015} Published online: 12 August 2015

\section{References}

1. Spencer FA, Meyer TE, Gore JM, Goldberg RJ. Heterogeneity in the management and outcomes of patients with acute myocardial infarction complicated by heart failure: the National Registry of Myocardial Infarction. Circulation. 2002;105(22):2605-10.

2. Fokkema ML, James SK, Albertsson P, Akerblom A, Calais F, Eriksson P, et al. Population trends in percutaneous coronary intervention: 20-year results from the SCAAR (Swedish Coronary Angiography and Angioplasty Registry). J Am Coll Cardiol. 2013;61(12):1222-30. doi:10.1016/j.jacc.2013.01.007.

3. Terkelsen CJ, Jensen LO, Tilsted HH, Trautner S, Johnsen SP, Vach W, et al. Health care system delay and heart failure in patients with ST-segment elevation myocardial infarction treated with primary percutaneous coronary intervention: follow-up of population-based medical registry data. Ann Intern Med. 201 1;155(6):361-7. doi:10.7326/0003-4819-155-6-201 109200-00004.

4. Pennell D. Myocardial salvage: retrospection, resolution, and radio waves. Circulation. 2006;113(15):1821-3. doi:10.1161/CIRCULATIONAHA.105.618942.

5. Botker HE, Kaltoft AK, Pedersen SF, Kim WY. Measuring myocardial salvage. (1755-3245 (Electronic)).

6. Wright J, Adriaenssens T, Dymarkowski S, Desmet W, Bogaert J. Quantification of myocardial area at risk with T2-weighted CMR: comparison with contrast-enhanced CMR and coronary angiography. JACC CardiovasC Imaging. 2009;2(7):825-31. doi:10.1016/j.jcmg.2009.02.011.

7. Fuernau G, Eitel I, Franke V, Hildebrandt L, Meissner J, de Waha S, et al. Myocardium at risk in ST-segment elevation myocardial infarction comparison of T2-weighted edema imaging with the MR-assessed endocardial surface area and validation against angiographic scoring. JACC Cardiovasc Imaging. 2011;4(9):967-76. doi:10.1016/j.jcmg.2011.02.023.

8. Giri S, Chung YC, Merchant A, Mihai G, Rajagopalan S, Raman SV, et al. T2 quantification for improved detection of myocardial edema. J Cardiovasc Magn Reson. 2009;11:56. doi:10.1186/1532-429X-11-56.

9. Verhaert D, Thavendiranathan P, Giri S, Mihai G, Rajagopalan S, Simonetti OP, et al. Direct T2 quantification of myocardial edema in acute ischemic injury. JACC Cardiovasc Imaging. 2011;4(3):269-78. doi:10.1016/j.jcmg.2010.09.023.
10. Thuny F, Lairez O, Roubille F, Mewton N, Rioufol G, Sportouch C, et al. Post-conditioning reduces infarct size and edema in patients with ST-segment elevation myocardial infarction. J Am Coll Cardiol. 2012:59(24):2175-81. doi:10.1016/j.jacc.2012.03.026.

11. White SK, Frohlich GM, Sado DM, Maestrini V, Fontana M, Treibel TA et al. Remote Ischemic Conditioning Reduces Myocardial Infarct Size and Edema in Patients With ST-Segment Elevation Myocardial Infarction. JACC Cardiovascular interventions. 2014. doi:10.1016/j.jcin.2014.05.015.

12. Crimi G, Pica S, Raineri C, Bramucci E, De Ferrari GM, Klersy C, et al. Remote ischemic post-conditioning of the lower limb during primary percutaneous coronary intervention safely reduces enzymatic infarct size in anterior myocardial infarction: a randomized controlled trial. JACC Cardiovasc Interv. 2013;6(10):1055-63. doi:10.1016/j.jcin.2013.05.011.

13. Ferreira VM, Piechnik SK, Dall'Armellina E, Karamitsos TD, Francis JM, Ntusi N, et al. T(1) mapping for the diagnosis of acute myocarditis using CMR: comparison to T2-weighted and late gadolinium enhanced imaging. JACC Cardiovasc Imaging. 2013;6(10):1048-58. doi:10.1016/j.jcmg.2013.03.008.

14. Dall'Armellina E, Piechnik SK, Ferreira VM, Si QL, Robson MD, Francis JM, et al. Cardiovascular magnetic resonance by non contrast T1-mapping allows assessment of severity of injury in acute myocardial infarction. J Cardiovasc Magn Reson. 2012;14:15. doi:10.1186/1532-429X-14-15.

15. Ugander M, Bagi PS, Oki AJ, Chen B, Hsu LY, Aletras AH, et al. Myocardial edema as detected by pre-contrast T1 and T2 CMR delineates area at risk associated with acute myocardial infarction. JACC Cardiovasc Imaging. 2012:5(6):596-603. doi:10.1016/j.jcmg.2012.01.016.

16. Langhans B, Nadjiri J, Jahnichen C, Kastrati A, Martinoff S, Hadamitzky M. Reproducibility of area at risk assessment in acute myocardial infarction by T1- and T2-mapping sequences in cardiac magnetic resonance imaging in comparison to Tc99m-sestamibi SPECT. Int J Cardiovasc Imaging. 2014;30(7):1357-63. doi:10.1007/s10554-014-0467-z.

17. von Knobelsdorff-Brenkenhoff F, Prothmann M, Dieringer MA, Wassmuth $R$, Greiser A, Schwenke C, et al. Myocardial T1 and T2 mapping at 3T: reference values, influencing factors and implications. J Cardiovasc Magn Reson. 2013:15:53. doi:10.1186/1532-429X-15-53.

18. White SK, Bulluck H, Frohlich GM, Casson SG, O'Meara C, Newton AC et al. Hybrid PET/MR metabolic imaging of the reperfused infarct - new biology, future directions. J Cardiovasc Magn Resonance. 2015; 17(Suppl 1):041. doi:10.1186/1532-429X-17-S1-O41.

19. Kellman P, Wilson JR, Xue H, Ugander M, Arai AE. Extracellular volume fraction mapping in the myocardium, part 1: evaluation of an automated method. J Cardiovasc Magn Reson. 2012;14:63. doi:10.1186/1532-429X-14-63.

20. Schulz-Menger J, Bluemke DA, Bremerich J, Flamm SD, Fogel MA, Friedrich MG, et al. Standardized image interpretation and post processing in cardiovascular magnetic resonance: Society for Cardiovascular Magnetic Resonance (SCMR) board of trustees task force on standardized post processing. J Cardiovasc Magn Reson. 2013;15:35. doi:10.1186/1532-429X-15-35.

21. Cerqueira MD, Weissman NJ, Dilsizian V, Jacobs AK, Kaul S, Laskey WK, et al. Standardized myocardial segmentation and nomenclature for tomographic imaging of the heart. A statement for healthcare professionals from the Cardiac Imaging Committee of the Council on Clinical Cardiology of the American Heart Association. Int J Cardiovasc Imaging. 2002;18(1):539-42.

22. Otsu N. A threshold selection method from gray-level histograms. IEEE Trans Syst Man Cyberb. 1979;9:62-6.

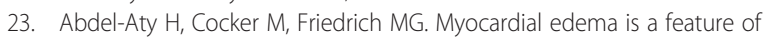
Tako-Tsubo cardiomyopathy and is related to the severity of systolic dysfunction: insights from T2-weighted cardiovascular magnetic resonance. Int J Cardiol. 2009;132(2):291-3. doi:10.1016/j.jjcard.2007.08.102.

24. DeLong ER, DeLong DM, Clarke-Pearson DL. Comparing the areas under two or more correlated receiver operating characteristic curves: a nonparametric approach. Biometrics. 1988;44(3):837-45.

25. Vermes E, Childs H, Carbone I, Barckow P, Friedrich MG. Auto-threshold quantification of late gadolinium enhancement in patients with acute heart disease. J Magn Reson Imaging. 2013;37(2):382-90. doi:10.1002/jmri.23814.

26. Kali A, Cokic I, Tang RL, Yang HJ, Sharif B, Marban E et al. Determination of location, size, and transmurality of chronic myocardial infarction without exogenous contrast media by using cardiac magnetic resonance imaging at 3T. (1942-0080 (Electronic)). doi:D - NLM: NIHMS580838 [Available on 05/01/15] D - NLM: PMC4077784 [Available on 05/01/15] OTO - NOTNLM.

27. Carlsson M, Ubachs JF, Hedstrom E, Heiberg E, Jovinge S, Arheden H. Myocardium at risk after acute infarction in humans on cardiac magnetic 
resonance: quantitative assessment during follow-up and validation with single-photon emission computed tomography. JACC Cardiovasc Imaging. 2009;2(5):569-76. doi:10.1016/j.jcmg.2008.11.018.

28. Dall'Armellina E, Ferreira VM, Kharbanda RK, Prendergast B, Piechnik SK

Robson MD, et al. Diagnostic value of pre-contrast T1 mapping in acute and chronic myocardial infarction. JACC Cardiovasc Imaging. 2013;6(6):739-42. doi:10.1016/j.jcmg.2012.11.020.

29. Fernandez-Jimenez R, Sanchez-Gonzalez J, Aguero J, Garcia-Prieto J,

Lopez-Martin GJ, Garcia-Ruiz JM et al. Myocardial Edema After Ischemia/ Reperfusion Is Not Stable and Follows a Bimodal Pattern: Advanced

Imaging and Histological Tissue Characterization. Journal of the American College of Cardiology. 2014. doi:10.1016/j.jacc.2014.11.004.

\section{Submit your next manuscript to BioMed Central and take full advantage of:}

- Convenient online submission

- Thorough peer review

- No space constraints or color figure charges

- Immediate publication on acceptance

- Inclusion in PubMed, CAS, Scopus and Google Scholar

- Research which is freely available for redistribution 